\title{
Bandwidth Enhancement of 5G Parallel Coupled Line Band Pass Filter Using Patterned Ground Structure Technique
}

\author{
Ummi Haziqah Morshidi ${ }^{1}$, Dyg Norkhairunnisa Abang Zaidel ${ }^{1^{*}}$, Norhudah \\ Seman $^{2}$, Melvin Philip Attan${ }^{1}$, Dayang Azra Awang Mat' ${ }^{1}$, Mohd Ridhuan Mohd \\ Sharip ${ }^{1}$, Haruichi Kanaya ${ }^{3}$
}

${ }^{1}$ Universiti Malaysia Sarawak, Kota Samarahan, 94300, Sarawak, MALAYSIA

${ }^{2}$ Universiti Teknologi Malaysia, Johor Bahru, 81110, Johor, MALAYSIA

${ }^{3}$ Kyushu University, Motooka 744, Fukuoka, JAPAN

*Corresponding Author

DOI: https://doi.org/10.30880/ijie.2020.12.06.011

Received 08 April 2020; Accepted 06 June 2020; Available online 02 July 2020

\begin{abstract}
This paper presents the design of parallel coupled line band pass filter with $10 \mathrm{GHz}$ operating frequency that will be used in 5G applications. As 5G application requires big data usage and to cater the applications, the bandwidth of $5 \mathrm{G}$ devices need to be wider enough to support it. Thus, to improve the bandwidth performance of the designed filter, patterned ground structure (PGS) technique is implemented into it. The result shows that the bandwidth of the designed filter has been improved from $0.25 \mathrm{GHz}$ to $4.98 \mathrm{GHz}$ when PGS is implemented.
\end{abstract}

Keywords: 5G application, Band Pass Filter, Bandwidth Enhancement, Parallel-Coupled, Patterned Ground Structure

\section{Introduction}

$5^{\text {th }}$ generation technology or known as $5 \mathrm{G}$ technology is a more advanced and powerful mobile technology that can support users with higher bandwidth and less significantly transmission delays. 5G network is expected to be launched in three to four years from now where the technology will meet revolution in enabling a host of new applications including humanoid robots, connected cars, and the Internet of Things (IoT) with its billions of devices laden with some embedded sensors [1]. The latest innovation of mobile data technology, the 5G will be as much as 1000 times faster than $4 \mathrm{G}$ technology with speeds of up to 100 gigabits per second [2].

The major facilitator for the ubiquity of smartphones is wireless communication system. To enable a future digital world that will transform a variety of economic sector, the overall $5 \mathrm{G}$ vision is going far beyond the evolution of mobile broadband. $5 \mathrm{G}$ as the modern wireless communication systems that are intended to provide the user with multiple services at ultrahigh data rates in a fully dynamic radio-access scenario [3]. IoT, self-driving cars with vehicle-to-vehicle and vehicle-to-everything connectivity are advancements in these systems which are playing a prominent role in realizing the vision.

The major breakthroughs such as long-term evolution and mm-wave standards are driven by these technologies in realms of electronics and packaging for multiband multi-standard communications. 5G wireless systems will use mmwave frequency bands, $28 \mathrm{GHz}$ U.S. $(24.5-29.5 \mathrm{GHz})$ and $39 \mathrm{GHz} \mathrm{EU}(37.0-43.5 \mathrm{GHz})$, to provide fast data rates of 100 $\mathrm{Mb} / \mathrm{s}$ to the end users in metropolitan areas [4]. However, as $5 \mathrm{G}$ is still under research state, thus there are still no specific operating frequency that is used for this technology. Based on findings, most of the $5 \mathrm{G}$ devices currently operated at 10 $\mathrm{GHz}, 15 \mathrm{GHz}$ and $28 \mathrm{GHz}$ [5-6]. Also, a 5G devices are considered under an operating frequency of $6 \mathrm{GHz}$ and above. 
The wireless communication industry is currently advancing to $5 \mathrm{G}$ which implies rapid growth of connectivity of many devices and a huge increase in mobile data rates. Thus, the networks are required to support 1000 times higher data volume per area, 10 to 100 times more connected devices in real time, and 10 to 100 times higher data rates [7]. The industry is focusing on innovative research to meet these demands into areas such as increased spectrum, improved efficiency and high-reliability communication links.

Spatial filters [8-12] are usually required in order to deal with stringent spectrum requirements. However, design of broadband passband spatial filters or frequency selective surface (FSS) filters can be very challenging due to the requirements of a wide passband and sharp transition from the passband edges to the stop-band [13].

As there are still lack of information and research on $5 \mathrm{G}$ device especially for passive devices, therefore, this paper will focus on the design of filter that will be used in $5 \mathrm{G}$ applications with operating frequency of $10 \mathrm{GHz}$. A parallel coupled microstrip line filter is designed and to improve the bandwidth's performance, patterned ground structure (PGS) is implemented into the designed filter.

\subsection{Parallel Coupled Line Band Pass Filter Design}

In radio frequency $(\mathrm{RF})$ front end of wireless communication systems, bandpass filters are the essential components especially in the future $5 \mathrm{G}$ massive multiple-input multiple-output (MIMO) system. Whereas, many bandpass filters are needed. RF and microwave bandpass filters play an important role in defining the operating bandwidth of a wireless or microwave system [14].

A lot of studies have been conducted to improve the performance and miniaturize the design of band pass filters. The most common method such as the multimode resonators (MMRs), folded quarter-wavelength resonator, or composite right/left-handed resonators are the methods to construct the compact bandpass filter [15].

Parallel coupled lines are widely used in microwave component designs, such as microstrip filters, delay lines, impedance matching networks, and directional couplers due to its easy incorporation in microwave-integrated circuits (MICs) [16]. Moreover, parallel coupled line filters are one of the most popular types of planar microwave bandpass filters [17]. Additionally, simple designs are often most practical and reliable.

To obtain a good filter design with required performance, design specification is needed to use as guidelines. It was chosen carefully to obtain the best results. Table 1 shows the design specification for the designed filter for this research. From Table 1, it can be observed that $10 \mathrm{GHz}$ is chosen as the operating frequency of the designed filter. Material used as the substrate is Rogers $4003 \mathrm{C}$ as it is the most suitable in the design of filters, matching networks and controlled the impedance transmission lines. Also, the dielectric constant and thickness for the Rogers 4003C are suitable for the condition of operating frequency at $10 \mathrm{GHz}$ [18].

The line impedance of the filter need to be $50 \Omega$ as it is called as impedance matching where all source, load impedance and transmission line is at their ideal condition to ensure zero or minimum power loss will be occurred during the signal transmission. Meanwhile, the return loss $\left(S_{1,1}\right)$, the loss of power in the signal that returned or reflected should be lower than $-10 \mathrm{~dB}$ which means that the reflected wave is $10 \mathrm{~dB}$ lower than the incident wave where upmost to $90 \%$ power is transmitted from input to output port. As for insertion loss $\left(S_{2,1}\right)$, the value should be approximately to $0 \mathrm{~dB}$ so that there is no loss of signal power resulting from the insertion of a device from input port.

\section{Design Specification}

All tables should be numbered with Arabic numerals. Every table should have a caption. Headings should be placed above tables, left justified. Only horizontal lines should be used within a table, to distinguish the column headings from the body of the table, and immediately above and below the table. Tables must be embedded into the text and not supplied separately. Below is an example which the authors may find useful.

Table 1 - Design Specification [19]

\begin{tabular}{cc}
\hline Specifications & Value \\
\hline Operating Frequency & $10 \mathrm{GHz}$ \\
Substrate & Rogers $4003 \mathrm{C}$ \\
$\left(\varepsilon_{r}=3.38\right)$ \\
Substrate Thickness & $0.508 \mathrm{~mm}$ \\
Line Impedance & $50 \Omega$ \\
Return loss $\left(\mathrm{S}_{1,1}\right)$ & $\leq-10 \mathrm{~dB}$ \\
Insertion loss $\left(\mathrm{S}_{2,1}\right)$ & $-0 \mathrm{~dB}$ \\
\hline
\end{tabular}




\subsection{Initial Design}

Each coupled section in $5 \mathrm{G}$ parallel coupled line band pass filter is defined by an input impedance $\left(Z_{I i}\right)$ and an input impedance ration $\left(\rho_{i}\right)$ [20]. Both of the input impedance and impedance ratio is defined as in equation (1) and equation (2).

$$
\begin{aligned}
& Z_{I i}=\sqrt{Z_{o e_{i}} Z_{o o_{i}}} ; i=1,2 \\
& \rho_{i}=\frac{Z_{o e_{i}}}{Z_{o o_{i}}}
\end{aligned}
$$

where $Z_{o e}$ and $Z_{o o}$ represents even and odd mode impedances of the coupled lines, respectively.

From equation (1), there are three possible input impedance $\left(Z_{i 1}\right)$ combination for two-port network that can be formed from a coupled line which shown from equation (3) to (5) [21].

$$
\begin{aligned}
& Z_{i 1}=\frac{2 Z_{0 e} Z_{0 o} \sin \theta}{\sqrt{\left(Z_{0 e}-Z_{0 o}\right)^{2}-\left(Z_{0 e}+Z_{0 o}\right)^{2} \cos ^{2} \theta}} \\
& Z_{i 1}=\frac{\sqrt{\left(Z_{0 e}-Z_{0 o}\right)^{2}-\left(Z_{0 e}+Z_{0 o}\right)^{2} \cos ^{2} \theta}}{2 \sin \theta} \\
& Z_{i 1}=\frac{\sqrt{Z_{0 e} Z_{0 o}} \sqrt{\left(Z_{0 e}-Z_{0 o}\right)^{2}-\left(Z_{0 e}+Z_{0 o}\right)^{2} \cos ^{2} \theta}}{\left(Z_{0 e}+Z_{0 o}\right) \sin \theta}
\end{aligned}
$$

In this paper, E-shaped patterned ground structure is introduced based on the T-shaped cell [22]. E-shaped structure has more improvement in terms of the sharpness of the transition knee and to reduce the circuit area.

Fig. 1 shows the even symmetry of common-mode signals transmission for the coupled E-shaped defected ground filter. From the figure, it is shown that the common signals passing through the coupled E-shaped patterned ground structure can be figured out as an ideal transmission line with even-mode characteristics $\left(Z_{e}\right)$ and a parallel LC resonator cascaded on the ground plane based on the even symmetry [22].

The gap capacitance between two sides of the slit and the equivalent inductance of the signal passing through the patterned ground structure can be denoted as $C_{p}$ and $L_{p}$, respectively. The extracted LC equivalent circuit is depicted in equation (6) and (7) [22].

$$
\begin{aligned}
& C_{p}=\frac{f_{c}}{4 \pi z_{e}}\left(\frac{1}{f_{o}^{2}-f_{c}^{2}}\right) \\
& L_{p}=\frac{1}{4 \pi^{2} f_{0}^{2} c_{p}}
\end{aligned}
$$

where, $f_{c}$ and $f_{o}$ are denoted as $3 \mathrm{~dB}$ cut-off frequency and the attenuation pole frequency of the band-stop response of the patterned ground structure resonator, respectively.

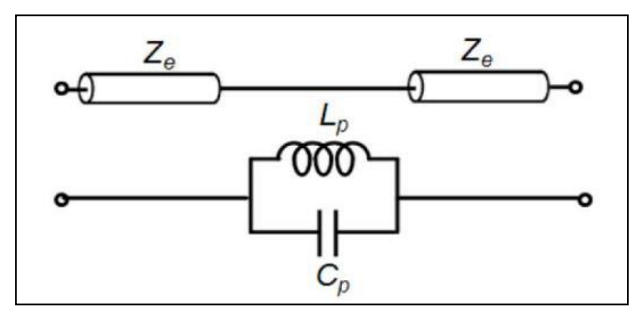

Fig. 1 - An even symmetry of common-mode signals transmission for the coupled e-shaped defected ground filter [23]

The equivalent circuits for coupled E-shaped resonators can be proven by the magnetic coupling coefficients of Ushaped resonator and $\mathrm{H}$-shaped resonated which are respectively denoted as $k_{m 1}$ and $k_{m 2}$ in equation (8) and (9) [22].

$$
k_{m 1}=\frac{L_{m 1}}{L_{1}}=\frac{f_{e}^{2}-f_{m}^{2}}{f_{e}^{2}+f_{m}^{2}}
$$


In equation (9), both $L_{1}$ and $C_{1}$ represent self-inductance and self-capacitance, respectively meanwhile $L_{m 1}$ is denoted as the mutual inductance where two split resonant frequencies are denoted as $f_{e}$ and $f_{m}$ due to the mutual coupling.

$$
k_{m 2}=\frac{L_{m 2}}{\sqrt{L_{1} L_{2}}}= \pm \frac{1}{2}\left(\frac{f_{o 2}}{f_{o 1}}+\frac{f_{o 1}}{f_{o 2}}\right) \times \sqrt{\left(\frac{f_{2}^{2}-f_{1}^{2}}{f_{2}^{2}+f_{1}^{2}}\right)^{2}-\left(\frac{f_{o 2}^{2}-f_{o 1}^{2}}{f_{o 2}^{2}+f_{o 1}^{2}}\right)^{2}}
$$

Meanwhile for equation (10), the $L_{2}$ and $C_{2}$ still represent both self-inductance and self-capacitance, respectively meanwhile $L_{m 2}$ is denoted as the mutual inductance where each resonator without mutual coupling for self-resonant frequencies are denoted respectively as $f_{o 1}$ and $f_{o 2}\left(f_{o 1}>f_{02}\right)$. The two-split resonant frequency due to the coupling are represents as $f_{1}$ and $f_{2}\left(f_{1}>f_{2}\right)$.

\section{Microstrip Design}

Based on the mathematical formulas and design specifications, the initial dimension of $5 \mathrm{G}$ parallel coupled line band pass filter was calculated and transformed into microstrip designed. Fig. 2 shows the 5G parallel coupled line band pass filter with the initial dimension based on the calculation. The design consists of 3 layers which are, the top patch, substrate in the middle and the bottom patch which act as the ground. In Fig. 2(b), the design of patch is fully black due to it is fully ground.

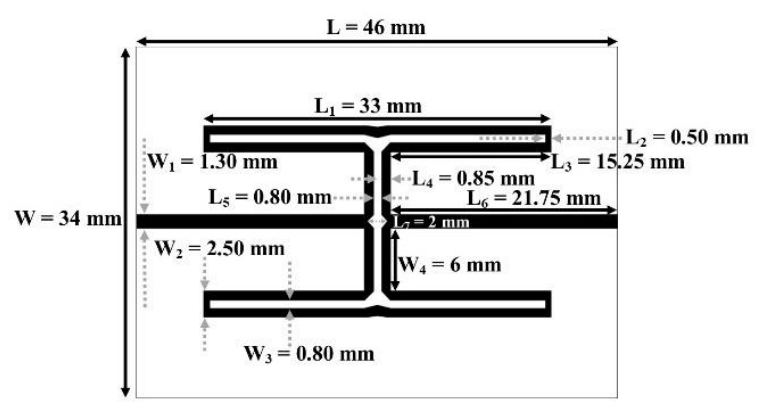

(a)

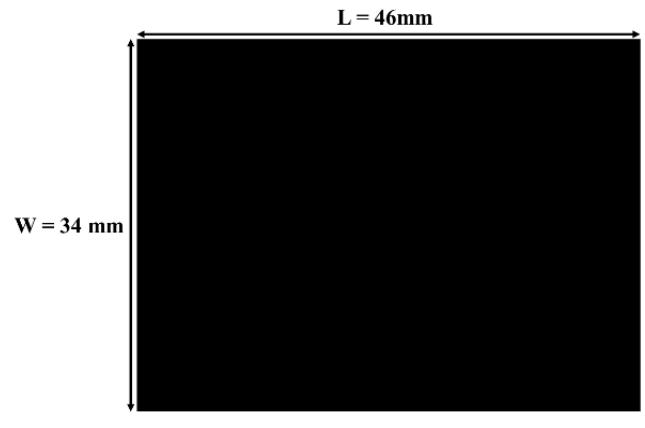

(b)

Fig. 2 - Initial design of $5 \mathrm{G}$ parallel coupled line band pass filter: (a) top patch; (b) ground patch

Then, the patterned ground structure (PGS) technique is applied into the initial 5G parallel coupled line band pass filter to observe the improvement on the bandwidth's performance. One of great advantage of this technique is its ability to enable the unwanted frequency rejection. Also PGS can improve the electrical performances and reduce the size of microstrip circuit. For this purpose, the front patch is remained as the initial design meanwhile the ground patch is applied with the PGS.

Fig. 3 depicts the design of the bottom section (ground patch) of the $5 \mathrm{G}$ parallel coupled line band pass filter with its parameter. As observed in Fig. 3, the resonant slot or gap is place directly under a transmission line. However, this slot must be aligned with the transmission line for efficient coupling to the transmission line in the ground metal.

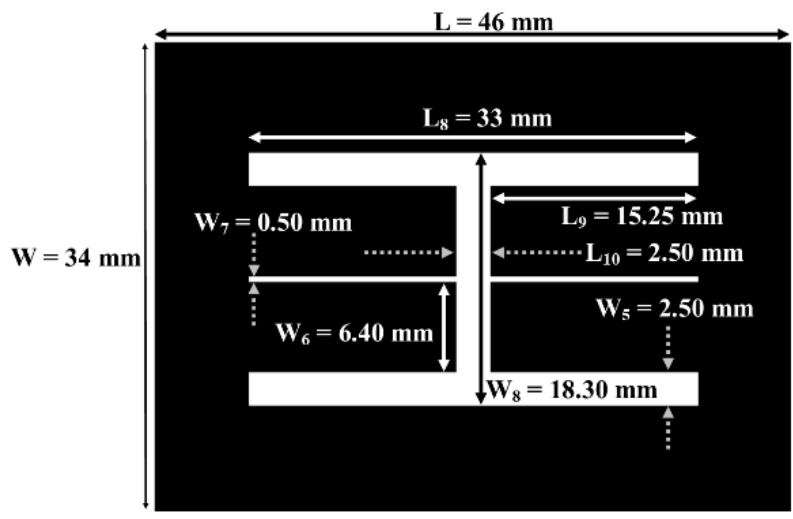

Fig. 3 - The design bottom section (ground patch) of the $5 \mathrm{G}$ parallel coupled line band pass filter 


\section{Results and Discussion}

Both of the designed filters were then simulate by using microwave Computer-Aided-Design (CAD) software. Fig. 4 shows the result of initial design $5 \mathrm{G}$ parallel coupled line band pass filter without the PGS implementation onto its ground. Based on the figure, it is observed that, at $10 \mathrm{GHz}$, the value of $S_{11}$ and $S_{21}$ are $-2.64 \mathrm{~dB}$ and $-4.47 \mathrm{~dB}$, respectively.

As seen, the initial result does not meet the requirement of design specification as mentioned earlier where the $S_{11}$ should be less than $-10 \mathrm{~dB}$ and $\mathrm{S}_{21}$ should be approximately to $0 \mathrm{~dB}$. In terms of bandwidth performance, the operating frequency is from $9.31 \mathrm{GHz}$ to $9.56 \mathrm{GHz}$ whereas resulting the value of bandwidth is only $0.25 \mathrm{GHz}$.

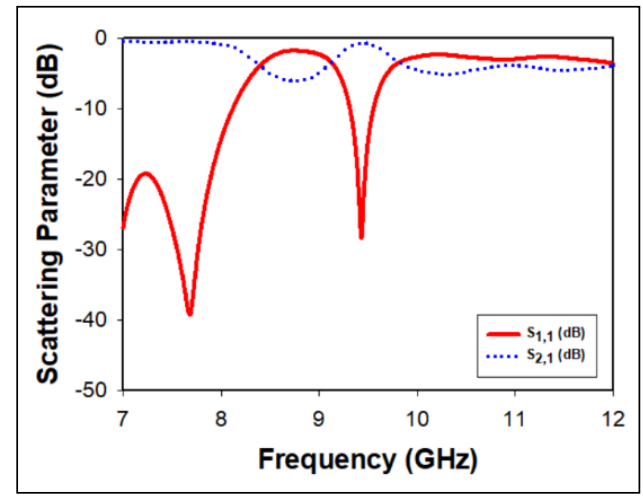

Fig. 4 - $S_{11}$ and $S_{21}$ simulation result of initial design (5G parallel coupled line band pass filter without the PGS implementation onto its ground patch)

Meanwhile, using the similar software, simulation is made towards the $5 \mathrm{G}$ parallel coupled line band pass filter with the PGS implementation onto its ground. Hence, Fig. 5 indicates its simulation result. As seen from the figure, it can be observed that there was improvement in the $S_{11}$ and $S_{21}$ where both of these losses marked at $-16.62 \mathrm{~dB}$ and $-1.76 \mathrm{~dB}$, respectively. Based on [24], it is acceptable for the value of insertion loss at approximately to $0 \mathrm{~dB}$ instead of precisely 0 $\mathrm{dB}$, where just small losses occurred from Port 1 to the Port 2. Meanwhile, for bandwidth, it is shown that the coverage is from $7 \mathrm{GHz}$ to $11.98 \mathrm{GHz}$ which is $4.98 \mathrm{GHz}$.

This result shows that by implementing the PGS onto its ground plane, the performance of the 5G parallel coupled line band pass filter is improved.

The PGS introduced the changes in the propagation of the wave along the line when there is placement of patterned ground structure resonators along the coupled line. This leads to the improvement.

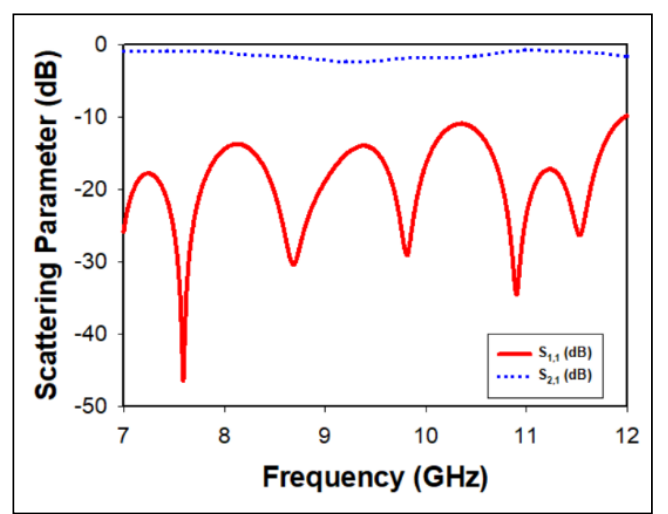

Fig. 5 - $S_{11}$ and $S_{21}$ simulation result of initial design (5G parallel coupled line band pass filter with the PGS implementation onto its ground patch)

Table 2 summarizes the comparison on the performances of both $5 \mathrm{G}$ parallel coupled line band pass filter without and with the PGS implementation onto its ground. Meanwhile, Fig. 6 shows the prototype of the designed filter after the fabrication process. 


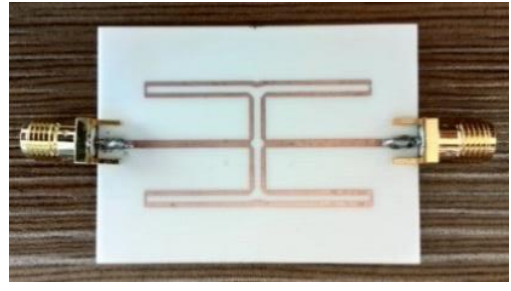

(a)

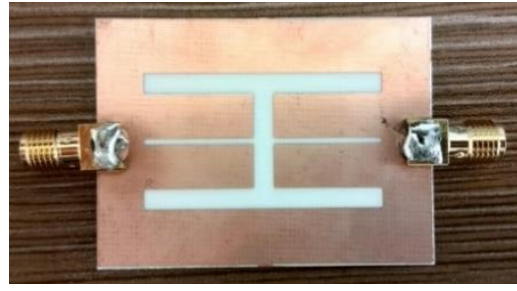

(b)

Fig. 6 -The prototype of 5G parallel coupled line band pass filter with PGS implementation: (a) front patch; (b) ground patch

Table 2 - Summary of the comparison on the performances of both 5G parallel coupled line band pass filter without and with the PGS implementation onto its ground

\begin{tabular}{ccc}
\hline Parameter & 5G Parallel Coupled Line Band Pass Filter & Value \\
without the PGS implementation & $\begin{array}{c}\text { 5G Parallel Coupled Line Band } \\
\text { Pass Filter with the PGS } \\
\text { implementation }\end{array}$ \\
\hline Return Loss, $\mathrm{S}_{11}(\mathrm{~dB})$ & -2.64 & -16.62 \\
Insertion Loss, $\mathrm{S}_{21}(\mathrm{~dB})$ & -4.47 & -1.76 \\
Operating Frequency & 9.31 to 9.56 & 7 to 11.98 \\
$(\mathrm{GHz})$ & 0.25 & 4.98 \\
\hline Bandwidth $(\mathrm{GHz})$ & & \\
\hline
\end{tabular}

\section{Conclusion}

In conclusion, the PGS technique has improved the performance of the $5 \mathrm{G}$ parallel coupled line band pass filter not limited to the bandwidth but also both return loss and also insertion loss. The bandwidth is improved from $0.25 \mathrm{GHz}$ to $4.98 \mathrm{GHz}$ which is up to $90 \%$ of improvement. The PGS will introduce the changes in the propagation of the wave along the line when there is placement of patterned ground structure resonators along the coupled line. However, this technique slows the even mode where it must propagate around the PGS slots, however it will not affect the odd mode transmission. This technique is good to be implemented as it helps in reducing the unwanted responses in the designed filter.

\section{References}

[1] K. Pretz. (2017). 5G : The Future of Communications Networks - IEEE - The Institute. The IEEE news source,. 2-3

[2] Infineon Technologies. (2019). 5G - The high-speed mobile network of the future - EE Times Asia. EET Asia

[3] Psychogiou D. and Gomez-Garcia R. (2017). Reflectionless Adaptive RF Filters: Bandpass, Bandstop, and Cascade Designs. IEEE Transaction on Microwave Theory and Technique. 65,11. 4593-4605

[4] Ali M. et al. (2018). First Demonstration of Compact, Ultra-Thin Low-Pass and Bandpass Filters for 5G Small-Cell Applications. IEEE Microwave and Wireless Components Letter. 28,12. 1110-1112

[5] Lee K., Choi S. and Kim C. (2019). A 25-30-GHz Asymmetric SPDT Switch for 5G Applications in 65-nm TripleWell CMOS. IEEE Microwave and Wireless Components Letters, 29, 6, 391-393

[6] Taheri M. M. S., Abdipour A., Zhang S. and Pedersen G. F. (2019). Integrated Millimeter-Wave Wideband EndFire 5G Beam Steerable Array and Low-Frequency 4G LTE Antenna in Mobile Terminals. IEEE Transactions on Vehicular Technology, 68, 4, 4042-4046

[7] Bangerter B., Talwar S., Arefi R. and Stewart K. (2014). Networks and Devices for the 5G Era. IEEE Communication Magazine. 52. 90-96

[8] Wu T.K. (2014). Improved Bandpass FSS for Wireless Communication. IEEE International Symposium on Antennas and Propagation and USNC-URSI Radio Science Meeting (APSURSI). Memphis, TN

[9] Ferreira D., Cuinas I., Caldeirinha R. and Fernandes T. (2016). Dual-Band Single Layer Quarter Ring FSS for WiFi Applications. IET Microwaves, Antennas \& Propagation. 10, 4. 435-441

[10] Wu T.K. (2017). Single-layer FSS for Wi-Fi applications. IEEE International Symposium on Antennas and Propagation and USNC-URSI Radio Science Meeting (APSURSI). San Diego, CA

[11]Liang E. and Wu T.K. (2017). Novel Wideband Frequency Selective Surface Filters with Fractal Elements. Microwave Journal. 60. 102-110

[12] Da Li, et al. (2017). A Low-Profile Broadband Bandpass Frequency Selective Surface with Two Rapid Band Edges for $5 \mathrm{G}$ Near-Field Applications. IEEE Transactions on Electromagnetic Compatibility. 59. 670-676

[13] Wu T. K. (2018). Improved Broadband Bandpass FSS Filters For 5G Applications. IEEE International Symposium on Antennas and Propagation and USNC-URSI Radio Science Meeting (APSURSI). 2033-2034 
[14]Chen C.-J. (2018). A Coupled-Line Coupling Structure for the Design of Quasi-Elliptic Bandpass Filters. IEEE Transaction on Microwave Theory and Technique. 66,4. 1921-1925

[15] Xu J. X. and Zhang X. Y. (2018). Dual-Channel Dielectric Resonator Filter and Its Application to Doherty Power Amplifier for 5G Massive MIMO System. IEEE Transaction on Microwave Theory and Technique. 66,7. 3297-3305

[16]Ding D., Zhang Q., Xia J., Zhou A., and Yang L. (2018). Wiggly Parallel-Coupled Line Design by Using Multiobjective Evolutionay Algorithm. IEEE Microwave and Wireless Components Letter. 28, 8. 648-650

[17] Chen C.J. (2016). Design of Parallel-Coupled Dual-Mode Resonator Bandpass Filters. IEEE Transaction on Components, Packaging and Manufacturing Technology. 6,10. 1542-1548

[18] Rogers Corporation Advanced Circuit Materials Division. (2006). RO4000 ® Series High Frequency Circuit Materials Data Sheet, 1-8

[19] Seman N. and Rahman T. A. (2015). Design of Microstrip Parallel-Coupled Line Band Pass Filters. Application in Fifth-Generation Wireless Communication. 9, 2, 19-23.

[20] S. E. L. Marini, J. Zbitou, R. Mandry, A. Errkik, and A. Tajmouati (2017). Beam Forming Network Application. 4, 3, 4-6

[21]D. Pozar, Microwave Engineering Fourth Edition. (2005). $3^{\text {rd }}$ Edition. Wiley and Sons. 426-436

[22]Zainol N., Zakaria Z., Abu M., Yunus M. M. and Ruslan E. (2017). Design of $2 \times 2$ array antenna with harmonic suppression using T-shape DGS and spurline. European Conference on Antennas and Propagation (EuCAP), 10441048.

[23] Wu S.J., Tsai C.H., Wu T.L, and Itoh T. (2009). A novel wideband common-mode suppression filter for gigahertz differential signals using coupled patterned ground structure. IEEE Transaction on Microwave Theory Technology, $57,4,848-855$

[24] Abbosh A.M. (2007). Ultra-wideband phase shifters. IEEE Transaction on Microwave Theory Technology, 55, 9, 1935-1941 